Artículo científico

Volumen 31(1):77-94. Enero-abril, 2020

e-ISSN 2215-3608, doi:10.15517/am.v31i1.36614

http://www.revistas.ucr.ac.cr/index.php/agromeso

\title{
Hojarasca e inóculo de Mycena citricolor sobre la epidemia de ojo de gallo ${ }^{1}$
}

\section{Leaf litter and Mycena citricolor inoculum on the American leaf spot epidemic}

\author{
Milagro Granados-Montero ${ }^{2}$, Jacques Avelino ${ }^{3}$, Felipe Arauz-Cavallini ${ }^{2}$, Sebastián Castro-Tanzi ${ }^{4}$, Natalia Ureña ${ }^{5}$
}

1 Recepción: 26 de marzo, 2019. Aceptación: 29 de agosto, 2019. Este trabajo formó parte de la tesis de doctorado en Sistemas de Producción Agrícola Tropical Sostenible de la primera autora. Financiado parcialmente por Earthwatch Institute, Massachusetts, Estados Unidos.

2 Universidad de Costa Rica, Escuela de Agronomía, Departamento de Protección de Cultivos. 2060 San José, Costa Rica. maria.granadosmontero@ucr.ac.cr (https://orcid.org/0000-0002-0321-7729), felipe.arauz@ucr.ac.cr

3 Centro de Cooperación Internacional en Investigación Agronómica para el Desarrollo (CIRAD). Montpellier, Francia. jacques.avelino@ cirad.fr (https://orcid.org/0000-0003-1983-9431).

4 Stone Environmental, Inc. Montpelier, Vermont, USA. scastro@stone-env.com

5 Centro Integral Simbiosis. Santa María de Dota, Costa Rica. urena.natalia@gmail.com

\section{Resumen}

Introducción. El ojo de gallo causado por Mycena citricolor está entre las principales enfermedades del cafeto en Costa Rica. Se presentan ataques cíclicos, aproximadamente cada catorce años, relacionados con el aumento de las precipitaciones y el inóculo. Objetivo. Determinar el impacto de la hojarasca y del inóculo primario sobre la epidemiología de esta enfermedad. Materiales y métodos. En cafetales ubicados en la región cafetalera de Tarrazú, Costa Rica, se valoró el efecto de la eliminación manual de las hojas enfermas adheridas a la planta y de la capa de hojas caídas, sobre la epidemia de ojo de gallo en los años 2013 y 2014. Se evaluaron 1200 bandolas en un experimento de parcelas divididas, donde los cuatro tratamientos se obtuvieron por la combinación de los niveles “con y sin" para cada factor. Se describió la enfermedad mediante la cuantificación del número de hojas enfermas, lesiones y geminíferos. Se construyeron las curvas de desarrollo de la enfermedad y se calculó el área bajo la curva para cada variable. Se calculó el crecimiento y defoliación de las plantas de café. Resultados. Con base en la curva de incidencia de la enfermedad para cada tratamiento en cada año, se determinó que el crecimiento logístico de las epidemias es el modelo de mejor ajuste. Las tasas de infección aparente ( $r$ ) fueron cercanas a 0,04 unidades por día en 2013 y no hubo diferencia estadística significativa en las epidemias desarrolladas; pero sí para el 2014, donde variaron entre 0,03 y 0,05 . Se observó un efecto principal del factor inóculo inicial sobre la presencia o ausencia de hojarasca. Conclusión. La hojarasca no tuvo efecto significativo en el desarrollo de la epidemia de ojo de gallo. Esta es la primera investigación que determina el impacto de posibles fuentes de inóculo distintas a las lesiones presentes en hojas de café.

Palabras clave: fitopatología, Coffea arabica, incidencia, enfermedades fungosas, Agaricomycetes.

\begin{abstract}
Introduction. The American leaf spot caused by Mycena citricolor is among the main coffee diseases in Costa Rica. Cyclic attacks occur approximately every fourteen years, related to increased rainfall and inoculum. Objective. Determine the impact of litterfall and primary inoculum on the epidemiology of this disease. Materials and methods.
\end{abstract}


In coffee plantations located in the coffee region of Tarrazú, Costa Rica, the effect of the manual elimination of diseased leaves attached to the plant and the layer of fallen leaves on the American leaf spot epidemic in 2013 and 2014 was assessed. 1200 branches were evaluated in an experiment of divided plots, where the four treatments were obtained by combining the "with and without" levels for each factor. The disease was described by quantifying the number of diseased leaves, lesions, and geminifers. The disease development curves were constructed and the area under the curve was calculated for each variable. The growth and defoliation of coffee plants was calculated. Results. Based on the disease incidence curve for each treatment in each year, it was determined that the logistic growth of epidemics is the best fit model. The rates of apparent infection $(r)$ were close to 0.04 units per day in 2013 and there was no significant statistical difference in the developed epidemics; but for 2014, where they varied between 0.03 and 0.05. A main effect of the initial inoculum factor was observed on the presence or absence of litterfall. Conclusion. Litterfall had no significant effect on the development of the American leaf spot epidemic. This is the first investigation that determines the impact of possible sources of inoculum other than the lesions present in coffee leaves.

Keywords: plant pathology, Coffea arabica, incidence, fungal diseases, Agaricomycetes.

\section{Introducción}

El ojo de gallo es una enfermedad causada por el hongo basidiomicete Mycena citricolor (Berk. \& M.A. Curtis) Sacc.; también se conoce como mancha americana de la hoja, gotera o candelilla, puede provocar pérdidas económicas relevantes en la producción de café por defoliación y eventual caída de frutos. Se reportó una infección máxima de $54 \%$, luego de períodos muy lluviosos en cafetales ubicados en una zona favorable para la enfermedad, en el norte de Guatemala; se estimó que con ese porcentaje de enfermedad se produjeron pérdidas de $56 \%$ de la producción (Avelino et al., 1995).

En Costa Rica se reportó el ojo de gallo desde 1876 (Wellman, 1951). Se presentan ataques cíclicos, aproximadamente cada catorce años, relacionados con el aumento de las precipitaciones y el inóculo (Carvajal 1939b; Borbón, 1999). En el año 2010 se presentó la última epidemia importante de ojo de gallo en café, para esa ocasión se contabilizó una disminución del $12 \%$ (alrededor de $71400000 \mathrm{~kg}$ ) de la cosecha estimada para el año productivo (2010-2011), lo que significó una pérdida de alrededor de \$60 millones USD (Barquero, 2010a; 2010b). Las zonas más afectadas fueron el Valle Central y la zona de Tarrazú, también llamada zona de Los Santos.

Entre 1995 y 1998, el ojo de gallo afectó alrededor de 3000 ha de cultivo, de las cuales 800 ha se ubicaban en la zona de Los Santos (Borbón, 1999). De acuerdo con la comisión técnica consultiva nacional del fenómeno ENOS del Instituto Meteorológico Nacional (COENOS, 2010) las condiciones climáticas de los años 1995, 1998 y 2010, fueron análogas y correspondieron con años de transición El Niño a La Niña.

El mayor efecto del ojo de gallo es la caída prematura de hojas (Carvajal, 1939a; Avelino et al., 1995; Wang y Avelino, 1999; Guerra, 2004; Barquero, 2007); esto produce una capa de hojarasca fresca constituida de follaje enfermo desprendido. Existe una correlación altamente significativa (76 \%) entre el índice de infección y el de defoliación (Avelino et al., 1995). Tanto la fase asexual (cuerpos geminíferos) como la sexual (basidiocarpos) de M. citricolor, han sido observados en hojas de diferentes especies vegetales caídas y en descomposición sobre el suelo, tanto en el bosque como en cultivos de cafeto (Carvajal, 1939a; 1939b; Buller, 1958; Dennis, 1961).

El género Mycena es uno de los predominantes dentro de las comunidades de agaricales saprófitos presentes en la hojarasca fresca y madera de los pisos de bosques húmedos tropicales. Estos hongos son colonizadores y descomponedores de hojas y madera, tanto en bosques como pasturas, ya que tienen la capacidad de degradar lignina y celulosa, por lo que su función típica es la de causar pudriciones blancas de la madera. Por ejemplo, $M$. 
galopus (Pers.) P. Kumm., es un descomponedor de hojas caídas de roble, su micelio puede abarcar el $80 \%$ de la hojarasca en estos bosques y puede permanecer en ella hasta por dos años. Muchas especies tienen la capacidad de producir metabolitos antifúngicos como las estrobirulinas, lo que les permite desplazar a otros hongos, algunos son capaces de vivir en el humus (Hibbett y Thorn, 2001; Moncalvo et al., 2002; Cannon y Kirk, 2007; Webster y Weber, 2007; Hernández, 2009). Mycena citricolor tiene una fase saprofítica, por tanto, existe la posibilidad de que se mantenga inóculo en la superficie del suelo del cafetal.

El ojo de gallo es más dependiente de la cantidad de inóculo primario que de la tasa de infección $(r)$, lo que supone que la implementación de estrategias de manejo que consigan reducir el nivel de inóculo inicial lograría retrasar considerablemente el desarrollo de la epidemia (Wang y Arauz, 1999), por ende, disminuir las pérdidas productivas y económicas. Este valor $(r)$ significa un aumento en la cantidad de inóculo o de enfermedad (incidencia o severidad), puede ser calculado por días, semanas o años; en general, el valor de $r$ para las enfermedades de ciclo múltiple es mayor que la $r\left(r_{m}\right)$ de enfermedades monocíclicas (Agrios, 2005). Con este valor se puede comparar entre epidemias, por ejemplo, desarrolladas en varios años o en diferentes condiciones; también permite comparar y relacionar entre los diferentes elementos y etapas de una epidemia, tales como inóculo primario, liberación de esporas, período latente, período infeccioso y otros (Achicanoy, 2000). Puede ser considerado como una medida del riesgo de una enfermedad y es uno de los tres parámetros epidemiológicos claves para desarrollar estrategias de manejo; los otros son la cantidad de inóculo inicial $\left(y_{0}\right)$ y el tiempo $(t)$ en que el patógeno y el hospedero interactúan. El valor de $r$ en enfermedades de varios ciclos es el que tiene mayor importancia para establecer pautas de manejo que logren reducir la enfermedad de manera agroecológica y económicamente sostenible (Nutter, 2007). Si la tasa de infección aparente es baja, la reducción en el nivel de enfermedad inicial retarda el desarrollo de la enfermedad (Arauz, 2011).

Para reducir el inóculo primario se debe conocer dónde está ubicado, determinar si el hongo sobrevive solo en las lesiones o como habitante en la hojarasca; así también, es necesario conocer el impacto de cada fuente de inóculo, para luego trabajar en estrategias que logren reducir al máximo la fuente más importante.

En algunos patosistemas como Phytophthora palmivora (E.J. Butler) E.J. Butler - cacao (Konan y Guest, 2002) y Venturia inaequalis (Cooke) G. Winter-manzana (Holb, 2006), la hojarasca es un componente importante en el desarrollo de la enfermedad. Por ejemplo, la incidencia de roña por $V$. inaequalis, se redujo entre 56-79 \% al realizar la estrategia sanitaria de eliminación de hojas caídas de árboles enfermos, lo que indica que en la hojarasca se mantiene el inóculo primario de la epidemia (Holb, 2006). En cacao ocurre lo contrario, ya que, si no existe capa de hojarasca, la enfermedad se ve favorecida, porque se disminuye la velocidad de descomposición de los residuos infectados; así, el patógeno sobrevive más tiempo en el campo y se aumenta el inóculo primario. Al respecto, Wellman (1951) indicó que M. citricolor (mencionada como Omphalia flavida Maubl. \& Rangel) tiene un corto período saprofítico en hojas podridas caídas de las plantas enfermas o cualquier otra materia en descomposición; también señaló que la diseminación de la enfermedad es lenta y difícil, siendo su único agente dispersor el salpique de la lluvia.

Se recomienda la deshoja de hojas enfermas como una estrategia de manejo adecuada para la enfermedad, y luego de este tratamiento las plantas pueden permanecer libres de ojo de gallo hasta por año y medio (Wellman, 1951; Plá, 1952).

Posterior a estas citas, la literatura no hace más referencia al uso de deshoja o al efecto de la capa de hojas en el piso del cafetal en relación con el desarrollo de la epidemia de ojo de gallo. El objetivo de esta investigación fue determinar el impacto de la hojarasca y del inóculo primario sobre la epidemiología de la enfermedad. 


\section{Materiales y métodos}

La investigación se realizó en los años 2013 y 2014 en la región cafetalera Tarrazú, la cual produce café que se comercializa en un $95 \%$ como estrictamente duro, Strictly Hard Bean (SHB) (ICAFE, 2019). Esta zona es considerada como una región lluviosa y mesotermal, por estar ubicada entre los 800 y $2000 \mathrm{msnm}$, con una lluvia anual entre 2000 y $4000 \mathrm{~mm}$ y una temperatura media anual entre 10 a $22^{\circ} \mathrm{C}$ (Solano y Villalobos, sf).

Se trabajó en cuatro fincas situadas en los cantones de Dota $\left(9^{\circ} 35^{\prime} 10^{\prime \prime} \mathrm{N}, 83^{\circ} 54^{\prime} 26^{\prime \prime} \mathrm{O}\right.$, altitud de $\left.1548 \mathrm{~m}\right)$ y Tarrazú $\left(9^{\circ} 40^{\prime} 0^{\prime}\right.$ N, $84^{\circ} 2^{\prime} 0^{\prime \prime}$, altitud de 1429 m); ambos situados en la provincia de San José, Costa Rica (Figura 1). Todas las plantas evaluadas fueron de Coffea arabica L. cultivar Catuaí, de más de diez años. Los ensayos se establecieron al final de la época seca y las evaluaciones iniciaron al inicio de la época lluviosa.

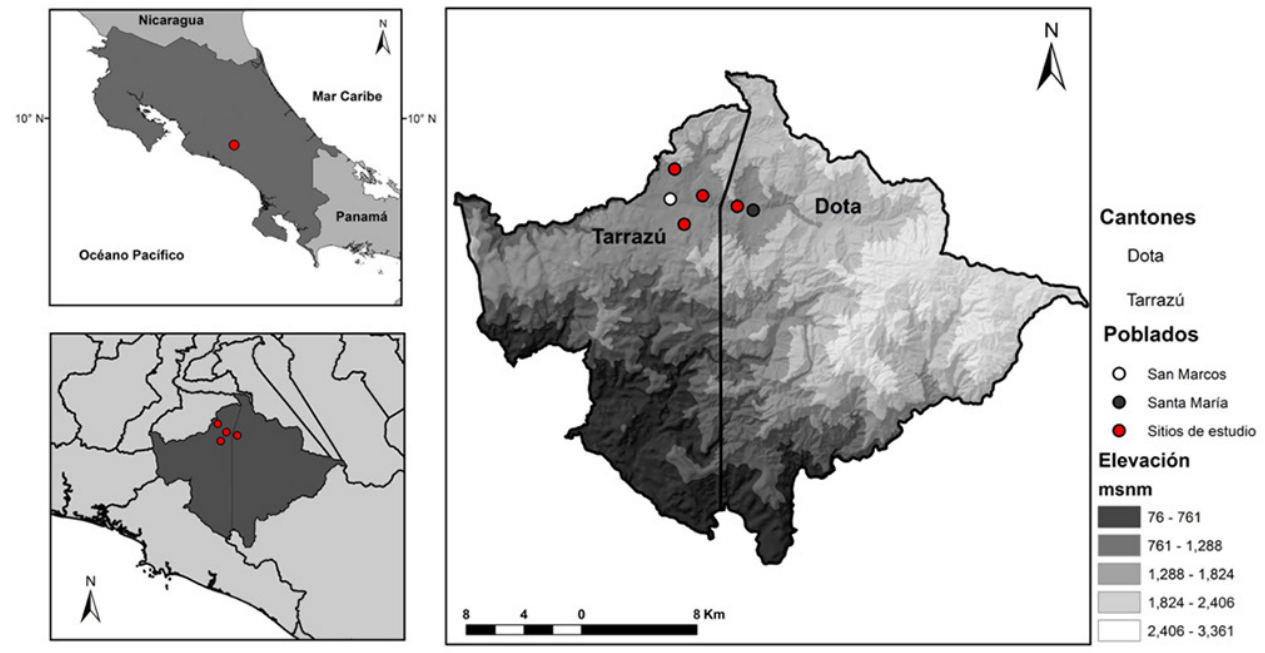

Figura 1. Ubicación de las fincas donde se determinó el impacto de la hojarasca y del inóculo primario sobre la epidemiología de ojo de gallo (Mycena citricolor) en café (Coffea arabica L. cultivar Catuaí) (sitios de estudio). Tarrazú, Costa Rica. 2013-2014.

Figure 1. Location of the farms where the impact of litterfall and primary inoculum on the epidemiology of American leaf spot (Mycena citricolor) in coffee (Coffea arabica L. cv Catuaí) (study sites) was determined. Tarrazú, Costa Rica. 2013-2014.

Se eligieron áreas de aproximadamente $400 \mathrm{~m}^{2}$ en cada finca, donde de acuerdo con la experiencia del productor o encargado, se había presentado históricamente la enfermedad ojo de gallo (M. citricolor) en similar incidencia. Para el establecimiento del ensayo del segundo año fue necesario cambiar la ubicación de dos bloques, debido a que las parcelas experimentales se podaron a profundidad, esto por mostrar severa defoliación a causa de la roya (Hemileia vastatrix Berk. \& Broome).

El diseño de los tratamientos fue de parcelas divididas con dos factores y dos niveles por factor. El primer factor, que representaba la parcela primaria, fue la hojarasca, denominada con la letra $\mathrm{H}$, este tenía dos niveles conforme a si se removió o no la capa de hojarasca presente en el suelo del cafetal. Así, el factor H, contó con los niveles "con hojarasca" (ConH), si no hubo remoción, y "sin hojarasca" (SinH), en el caso que se removió. La parcela secundaria fue el inóculo primario, nombrada con las letras IP. Este factor estuvo compuesto por los niveles "con inóculo primario" (ConIP), cuando no se eliminaron las hojas con lesiones, y "sin inóculo primario" (SinIP), cuando se eliminaron las hojas enfermas (Figura 2). 


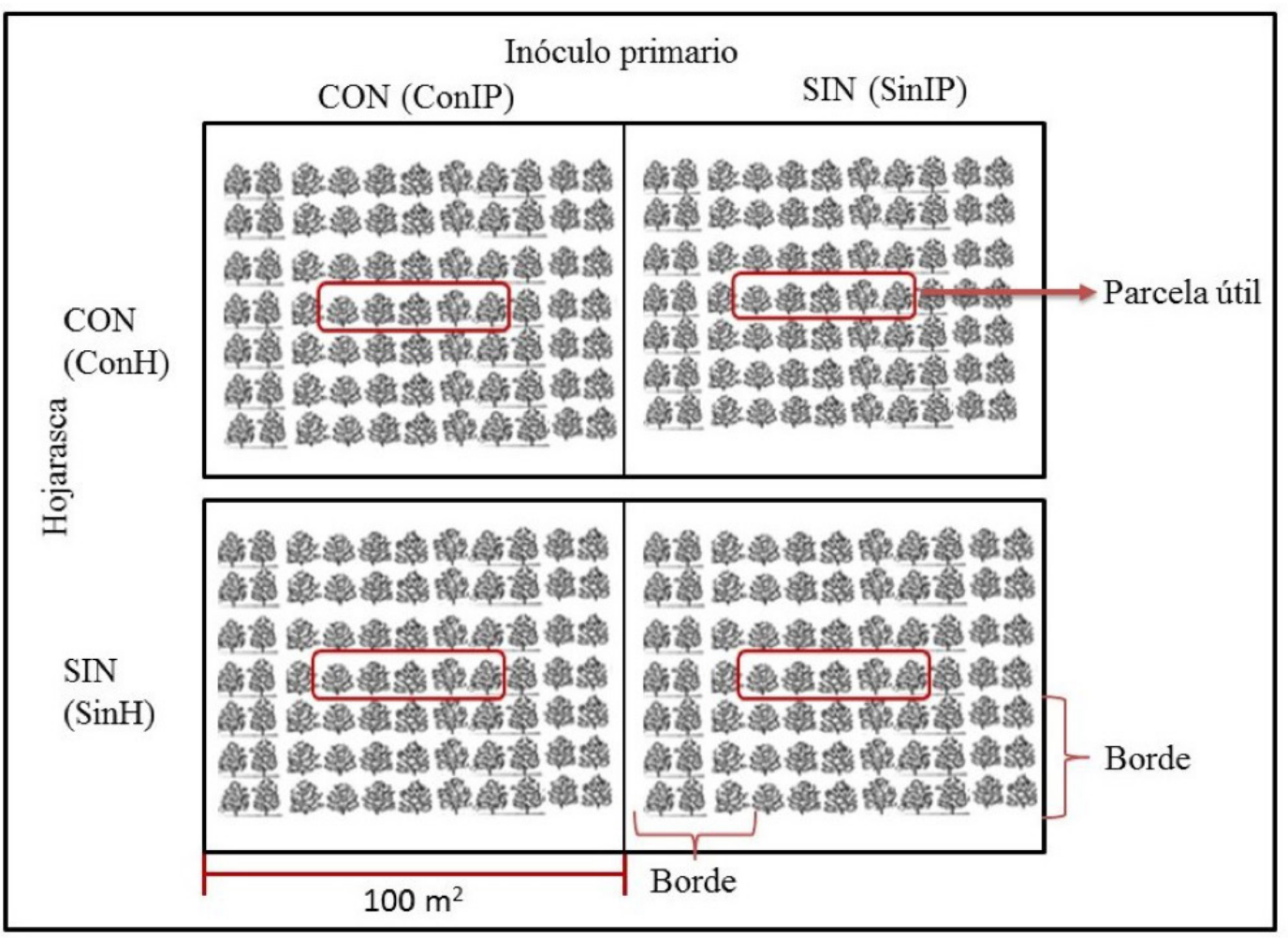

Figura 2. Diagrama del arreglo de tratamientos por bloque para determinar el impacto de la hojarasca y del inóculo primario sobre la epidemiología del ojo de gallo (Mycena citricolor) en café (Coffea arabica L. cultivar Catuaí). Tarrazú, Costa Rica. $2013-2014$.

ConH: con hojarasca; SinH: sin hojarasca; ConIP: con inóculo primario; SinIP: sin inóculo primario.

Figure 2. Diagram of the treatment arrangement per block to determine the impact of litterfall and primary inoculum on the epidemiology of American leaf spot (Mycena citricolor) in coffee (Coffea arabica L. cv Catuaí). Tarrazú, Costa Rica. $2013-2014$.

ConH: with litterfall; SinH: without litterfall; ConIP: with primary inoculum; SinIP: without primary inoculum.

El tratamiento ConH/ConIP en el que no se removió la hojarasca ni el inóculo primario, fue el que representó el manejo tradicional de la plantación (tratamiento testigo) en el ensayo. Todas las plantas se mantuvieron bajo el manejo nutricional rutinario de las fincas, el cual incluyó la aplicación al suelo de enmiendas con cal dolomita y tres fertilizaciones con las fórmulas cafetaleras, 18-5-15-6-2, 19-4-19-2, 14-4-23 o 15-1,5-25, en ese orden. Además, aplicación foliar de calcio, zinc, boro, magnesio, hierro y molibdeno.

La eliminación del inóculo de las plantas se hizo por medio de defoliación manual de todas las hojas que presentaban lesiones, ya fueran del año anterior o nuevas. La remoción de la capa de hojarasca se realizó cada catorce días, para mantener la parcela en condición de suelo desnudo (Figura 3). Las hojas enfermas y la capa de hojarasca se colocaron en zonas acondicionadas para tal efecto, fuera del área de estudio. Se les aplicó fungicida de acuerdo con la frecuencia de aplicación normal de las fincas.

El diseño experimental fue de bloques completos al azar, con seis bloques y cuatro tratamientos. La parcela útil fue de cinco plantas y el borde de tres. Se evaluaron cinco ramas plagiotrópicas primarias por planta, distribuidas al azar y que cubrían todos los estratos de la planta, para un total de 600 ramas evaluadas por año. 


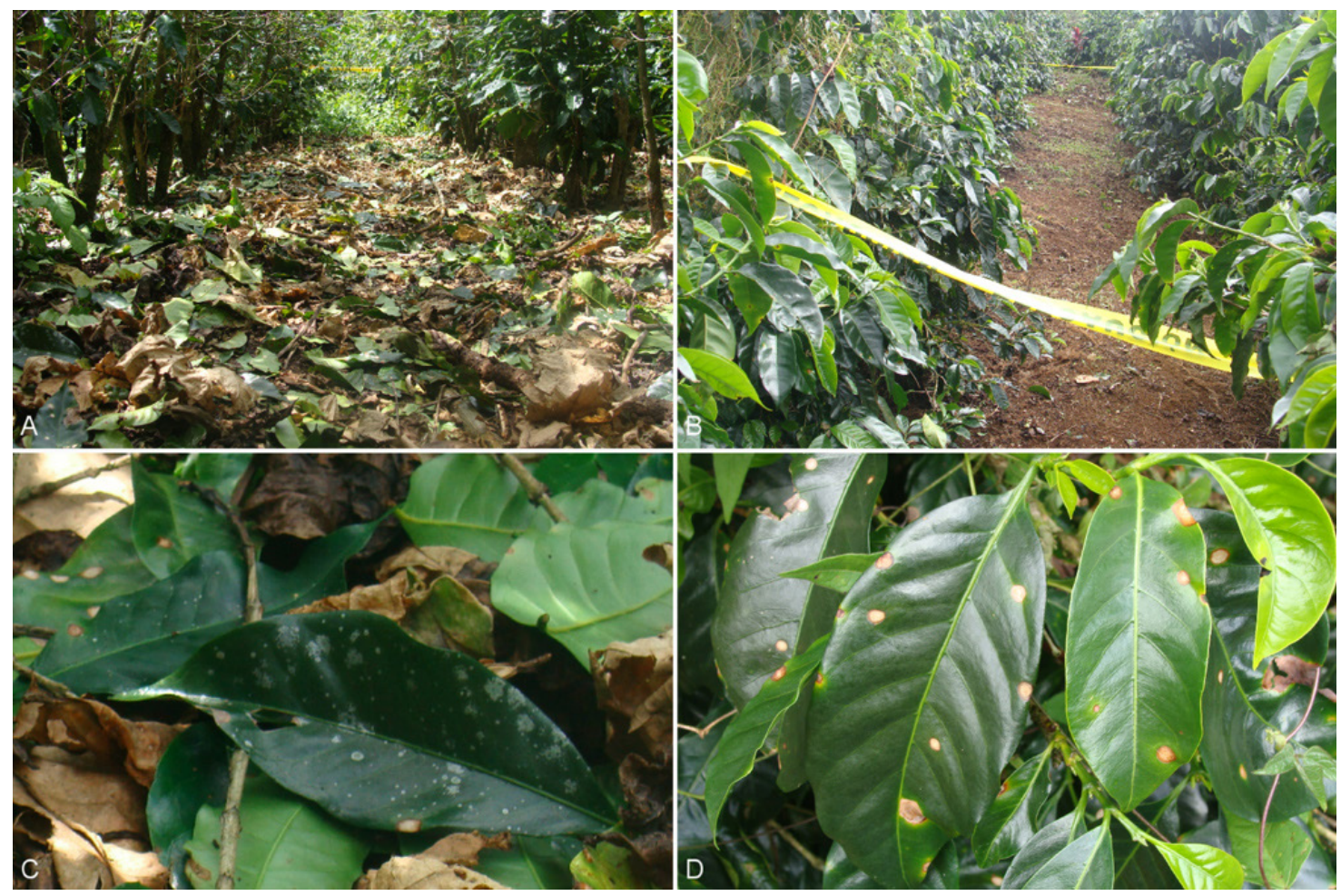

Figura 3. Ilustración de tratamientos para determinar el impacto de la hojarasca y del inóculo primario sobre la epidemiología del ojo de gallo (Mycena citricolor) en café (Coffea arabica L. cultivar Catuaí). Tarrazú, Costa Rica. 2013-2014.

A. Sin remoción de hojarasca. B. Con remoción de hojarasca. C. Detalle de la hojarasca. D. Detalle de inóculo primario.

Figure 3. Illustration of treatments to determine the impact of litterfall and primary inoculum on the epidemiology of American leaf spot (Mycena citricolor) in coffee (Coffea arabica L. cultivar Catuaí). Tarrazú, Costa Rica. 2013-2014.

A. Without litterfall removal. B. With removal of litterfall. C. Detail of litterfall. D. Detail of primary inoculum.

Cada catorce días se cuantificó, en la zona de nuevo crecimiento de las ramas, las siguientes variables: cantidad de hojas por nudo, cantidad de hojas enfermas, cantidad de lesiones en cada hoja y cantidad de geminíferos activos ${ }^{5}$ en el haz de cada hoja. Con los datos se calculó, para cada evaluación, las cantidades presentes, nuevas y caídas de: hojas sanas, hojas enfermas, lesiones y geminíferos; adicionalmente, se obtuvo el porcentaje acumulado de crecimiento del hospedero (rama) y de defoliación, y el porcentaje acumulado final de la enfermedad (AFE), corregido por el crecimiento del hospedero, para describir las epidemias en cada año.

$$
A F E=100 *(\text { Dact } / \text { HTacf })
$$

Donde:

Dact $=$ incidencia de la enfermedad expresada como hojas enfermas o lesiones acumuladas en el tiempo t.

$\mathrm{t}=$ fecha de evaluación.

5 Geminíferos erectos de color amarillo brillante, no se contabilizaron los deshidratados o inmaduros. 
HTacf $=$ hojas totales acumuladas en el tiempo $\mathrm{f}$.

$\mathrm{f}=$ tiempo final= última evaluación.

Luego, se calculó el área bajo la curva de desarrollo de la enfermedad (ABCDE).

$$
A B C D E=\sum^{n-1}((y i+y i+1) / 2) *(t i+1-t i)
$$

Donde:

$\mathrm{n}=$ número de evaluaciones.

$y=$ cantidad de la enfermedad.

$\mathrm{t}=$ tiempo en días.

Se usaron las curvas de incidencia para determinar el modelo de mejor ajuste de crecimiento de las epidemias (logístico, monomolecular o Gompertz) en cada tratamiento, en cada año. Se linealizaron las curvas con el parámetro $K$ para corregir la proporción de enfermedad máxima, una vez transformada la variable, se procedió a realizar un análisis de regresión lineal para determinar la tasa de infección aparente $(r)$ de cada epidemia para cada modelo.

Se realizó un $\mathrm{ANDEVA}^{6}$ para determinar si existían diferencias entre bloques, factores e interacción de factores (tratamientos) para las variables: 1) acumulado final de hojas enfermas, 2) acumulado final de lesiones, 3) acumulado final de geminíferos, 4) promedio de lesiones por hoja, 5) promedio de geminíferos por lesión, 6) porcentaje de lesiones con geminíferos, 7) hojas enfermas totales, 8) lesiones totales, 9) geminíferos totales, 10) crecimiento del hospedero, 11) defoliación del hospedero y 12) defoliación de hojas enfermas. Luego se realizó la prueba de comparación de medias LSD de Fischer al $95 \%$ de confianza, para determinar la magnitud de las diferencias.

Los datos se analizaron por medio de los programas de análisis estadísticos Infostat versión 2015 (Di-Rienzo et al., 2015) y RStudio (versión 0.99.473-2009-2015). Las gráficas se realizaron con la opción de gráficos de Excel de Microsoft Office 2013.

Durante la prueba se registraron los valores de temperatura, humedad relativa, precipitación y período de mojadura foliar, por medio del colector de datos meteorológicos "WatchDog 1000 series".

Se realizó una matriz de correlación mediante la técnica bivariada de Spearman entre las condiciones ambientales y las variables evaluadas; con confiabilidades de 95 y $99 \%$.

\section{Resultados}

El comportamiento de las variables climáticas durante los años de estudio se presenta en el Cuadro 1 y en las Figuras 4 y 5 .

Se muestra el acumulado final de lluvia, así como los mínimos y máximos de temperatura. Además, las fluctuaciones en temperatura, humedad relativa, precipitación y mojadura foliar para ambos años de estudio.

El ojo de gallo presentó baja incidencia en ambos años de estudio; para la época lluviosa 2013 tuvo un máximo cercano al $25 \%$, mientras que en el 2014 fue cercana al $20 \%$. Los tratamientos no fueron diferentes al testigo en el primer año de evaluación (Figura 6), pero sí se cuantificó efecto para el segundo año.

En el 2014, los tratamientos con inóculo primario fueron los que presentaron los valores más altos de incidencia acumulada durante todas las evaluaciones para ese año; el tratamiento SinH/ConIP llegó a una incidencia máxima de $19,30 \%$ y el tratamiento ConH/ConIP a 15,79 \%. Los tratamientos de eliminación de inóculo, ConH/SinIP y SinH/SinIP, presentaron menor incidencia $10,90 \%$ y 10,30 \%, respectivamente (Figura 7).

\footnotetext{
6 Se evaluó la homogeneidad y normalidad de las variables previo al ANDEVA.
} 
Cuadro 1. Temperaturas $\left({ }^{\circ} \mathrm{C}\right)$ mínima, máxima, y precipitación acumulada en los años de estudio para determinar el impacto de la hojarasca y del inóculo primario sobre la epidemiología del ojo de gallo (Mycena citricolor) en café (Coffea arabica L. cultivar Catuaí). Tarrazú, Costa Rica. 2013-2014.

Table 1. Minimum and maximum temperatures $\left({ }^{\circ} \mathrm{C}\right)$ and accumulated precipitation in the study years to determine the impact of litterfall and primary inoculum on the epidemiology of American leaf spot (Mycena citricolor) in coffee (Coffea arabica L. cultivar Catuaí). Tarrazú, Costa Rica. 2013-2014.

\begin{tabular}{|c|c|c|c|}
\hline \multirow[b]{2}{*}{ Año } & \multicolumn{2}{|c|}{ Temperatura $\left({ }^{\circ} \mathbf{C}\right)$} & \multirow[b]{2}{*}{ Precipitación (mm) } \\
\hline & Mínima & Máxima & \\
\hline 2013 & 14,2 & 22,3 & 1527 \\
\hline 2014 & 16,0 & 22,3 & 1997 \\
\hline
\end{tabular}

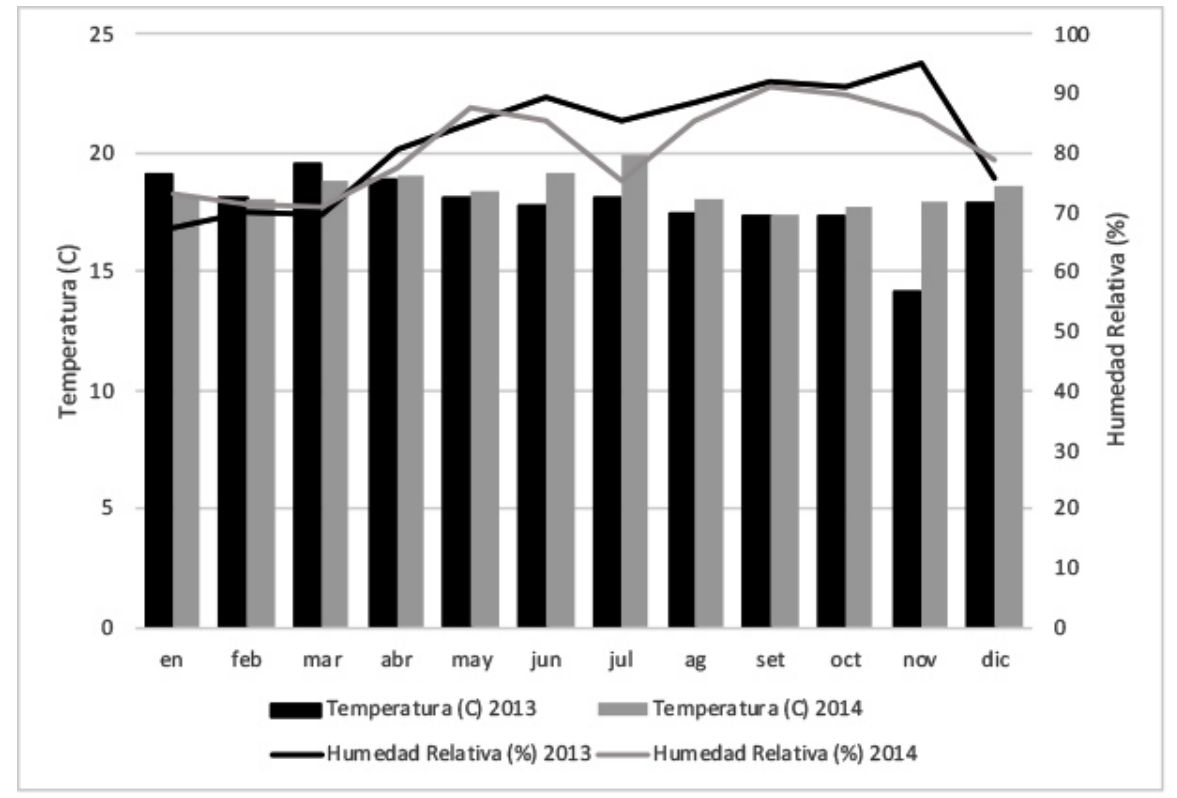

Figura 4. Fluctuación de temperatura $\left({ }^{\circ} \mathrm{C}\right)$ y humedad relativa $(\%)$ del área de estudio, para determinar el impacto de la hojarasca y del inóculo primario sobre la epidemiología del ojo de gallo (Mycena citricolor) en café (Coffea arabica L. cultivar Catuaí). Tarrazú, Costa Rica. 2013-2014.

Figure 4. Temperature fluctuation $\left({ }^{\circ} \mathrm{C}\right)$ and relative humidity $(\%)$ of the study area to determine the impact of litterfall and primary inoculum on the epidemiology of American leaf spot (Mycena citricolor) in coffee (Coffea arabica L. cultivar Catuaí). Tarrazú, Costa Rica. 2013-2014.

El tratamiento SinH/SinIP fue el que presentó el mayor valor de área bajo la curva de desarrollo de hojas enfermas, lesiones y geminíferos activos. No se encontraron diferencias significativas entre los valores calculados para cada tratamiento en el año epidemiológico 2013 (Cuadro 2).

Para el año 2014, la combinación SinH/ConIP fue la que presentó mayor valor de ABCDE para las variables hojas enfermas y lesiones; los geminíferos se encontraron en mayor cantidad en el tratamiento ConH/ConIP (Cuadro 2). 


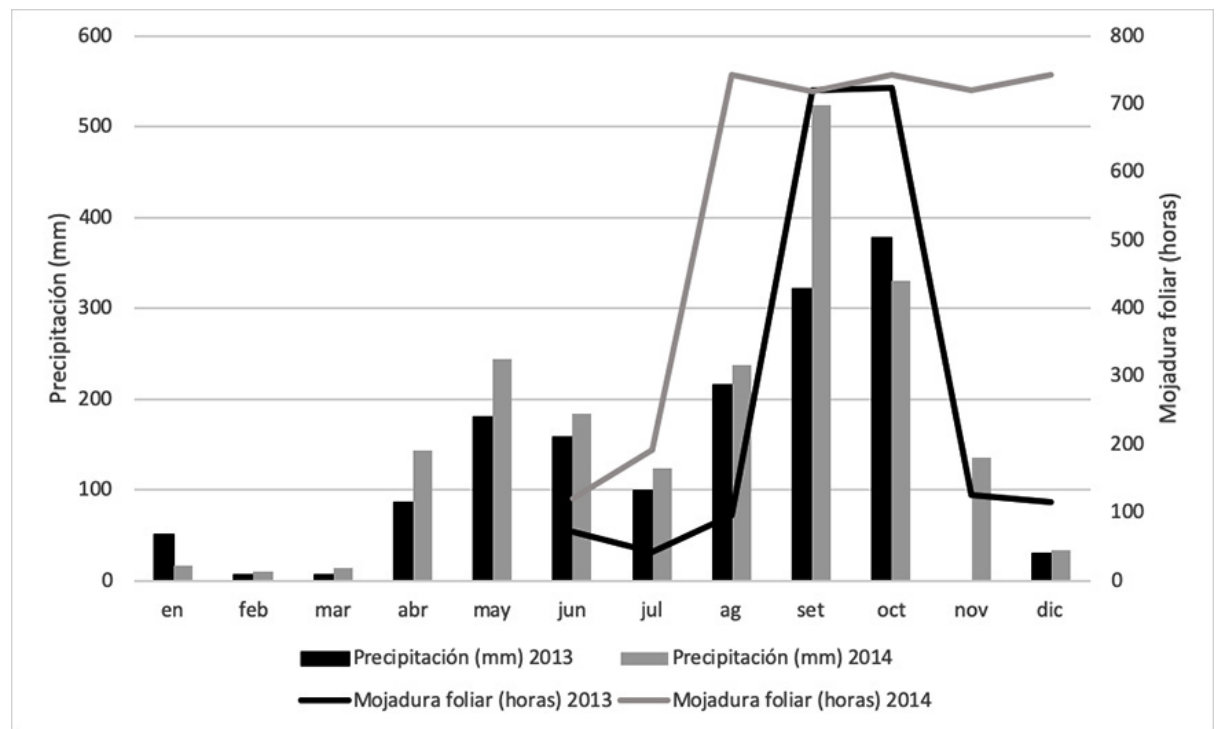

Figura 5. Condiciones de precipitación $(\mathrm{mm})$ y mojadura foliar (horas) del área de estudio para determinar el impacto de la hojarasca y del inóculo primario sobre la epidemiología del ojo de gallo (Mycena citricolor) en café (Coffea arabica L. cultivar Catuaí). Tarrazú, Costa Rica. 2013-2014.

Figure 5. Precipitation conditions ( $\mathrm{mm}$ ) and foliar wetting (hours) of the study area to determine the impact of litterfall and primary inoculum on the epidemiology of American leaf spot (Mycena citricolor) in coffee (Coffea arabica L. cultivar Catuaí). Tarrazú, Costa Rica. 2013-2014.

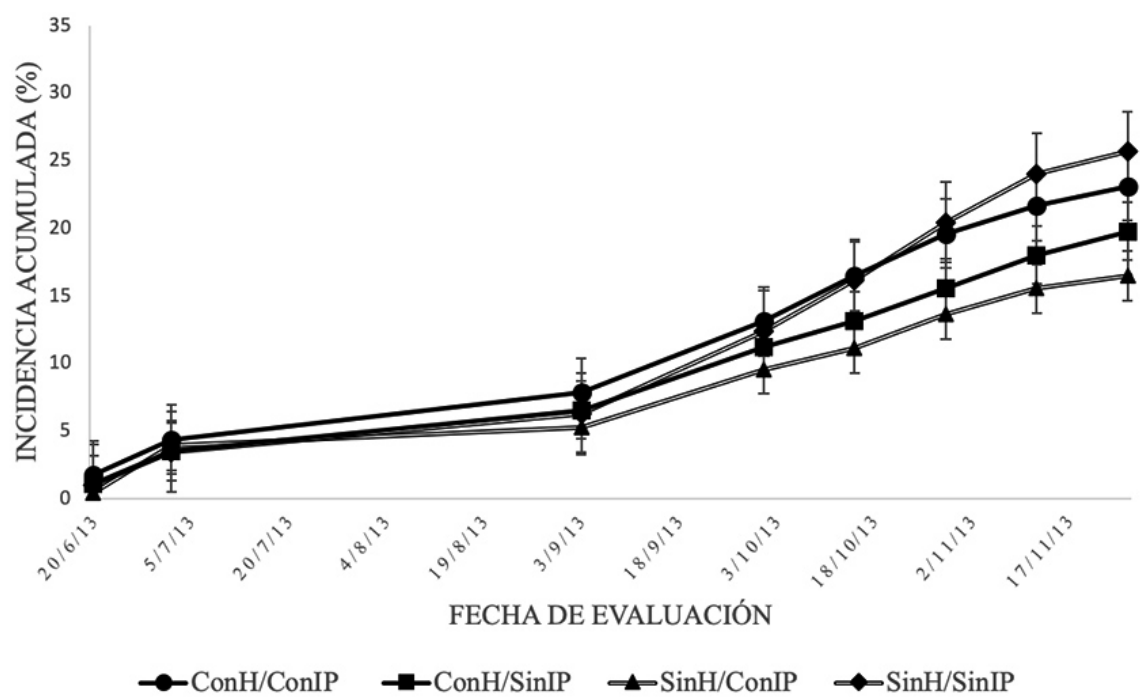

Figura 6. Curva de desarrollo del ojo de gallo (Mycena citricolor) en café (Coffea arabica L. cultivar Catuaí), expresada como incidencia acumulada (\%) de hojas con lesiones. Tarrazú, Costa Rica. 2013.

ConH: con hojarasca; SinH: sin hojarasca; ConIP: con inóculo primario; SinIP: sin inóculo primario.

Figure 6. American leaf spot (Mycena citricolor) development curve in coffee (Coffea arabica L. cultivar Catuaí), expressed as cumulative incidence (\%) of leaves with lesions. Tarrazú, Costa Rica. 2013.

ConH: with litterfall; SinH: without litterfall; ConIP: with primary inoculum; SinIP: without primary inoculum. 


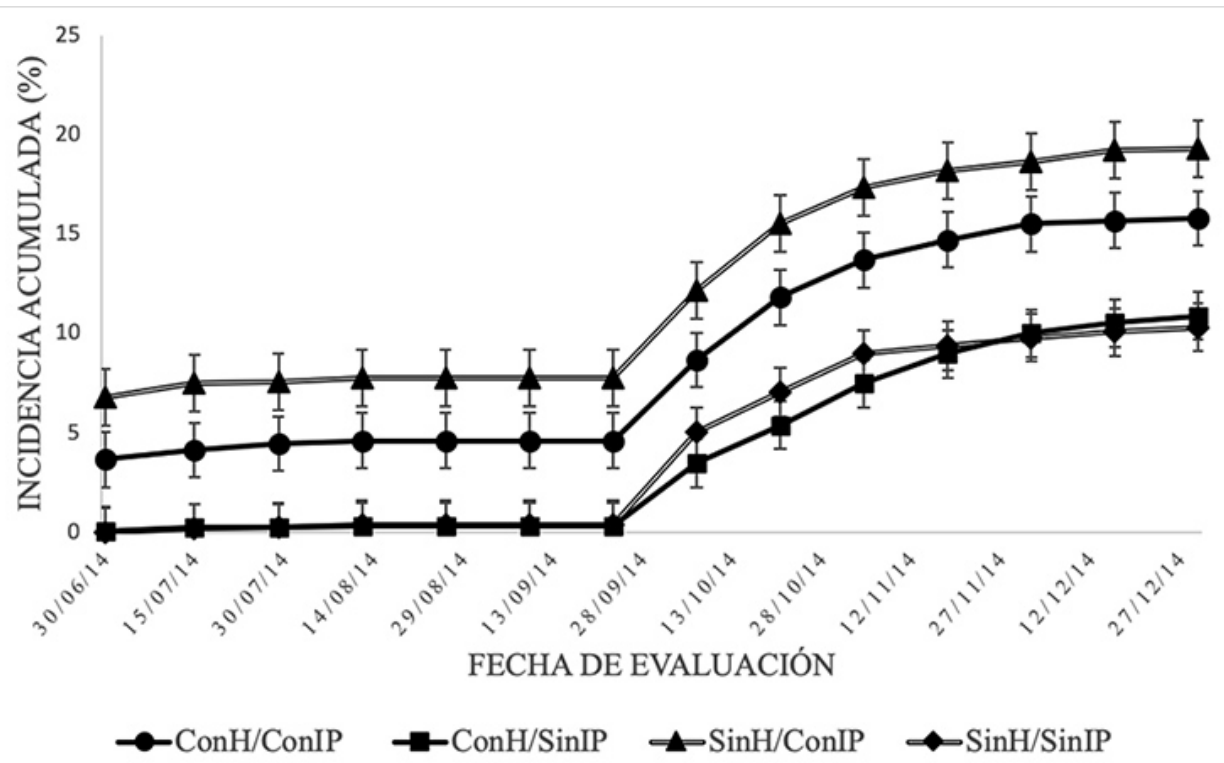

Figura 7. Desarrollo del ojo de gallo (Mycena citricolor) en café (Coffea arabica L. cultivar Catuaí), expresado como incidencia acumulada (\%). Tarrazú, Costa Rica. 2014.

ConH: con hojarasca; SinH: sin hojarasca; ConIP: con inóculo primario; SinIP: sin inóculo primario.

Figure 7. American leaf spot (Mycena citricolor) development in coffee (Coffea arabica L. cultivar Catuaí), expressed as cumulative incidence (\%). Tarrazú, Costa Rica. 2014.

ConH: with litterfall; SinH: without litterfall; ConIP: with primary inoculum; SinIP: without primary inoculum.

Cuadro 2. Área bajo la curva de desarrollo del ojo de gallo (Mycena citricolor) en café (Coffea arabica L. cultivar Catuaí), de acuerdo con el porcentaje acumulado final de hojas enfermas, lesiones y geminíferos activos. Tarrazú, Costa Rica. 2013-2014.

Table 2. Area under the development curve of American leaf spot (Mycena citricolor) development in coffee (Coffea arabica L. cultivar Catuaí), according to the final accumulated percentage of diseased leaves, lesions and active geminifers. Tarrazú, Costa Rica. 2013-2014.

\begin{tabular}{lcccccc}
\hline Tratamiento & \multicolumn{5}{c}{ Área bajo la curva de desarrollo de la enfermedad } \\
\cline { 2 - 6 } & \multicolumn{5}{c}{$\mathbf{2 0 1 3}$} & \multicolumn{2}{c}{$\mathbf{2 0 1 4}$} \\
\cline { 2 - 6 } & Hojas enfermas & Lesiones & $\begin{array}{c}\text { Geminíferos } \\
\text { activos }\end{array}$ & Hojas enfermas & $\begin{array}{c}\text { Lesiones } \\
\text { Geminíferos } \\
\text { activos }\end{array}$ \\
\hline ConH/ConIP & $1784,37^{\mathrm{a}}$ & $4542,80^{\mathrm{a}}$ & $1242,50^{\mathrm{a}}$ & $1642,44^{\mathrm{ab}}$ & $5595,79^{\mathrm{ab}}$ & $4075,71^{\mathrm{ab}}$ \\
ConH/SinIP & $1482,23^{\mathrm{a}}$ & $3672,82^{\mathrm{a}}$ & $2237,67^{\mathrm{a}}$ & $745,00^{\mathrm{a}}$ & $2246,30^{\mathrm{a}}$ & $935,28^{\mathrm{a}}$ \\
SinH/ConIP & $1287,88^{\mathrm{a}}$ & $5321,24^{\mathrm{a}}$ & $5748,17^{\mathrm{a}}$ & $2252,09^{\mathrm{b}}$ & $8565,84^{\mathrm{b}}$ & $3065,90^{\mathrm{b}}$ \\
SinH/SinIP & $1803,86^{\mathrm{a}}$ & $6074,72^{\mathrm{a}}$ & $8503,83^{\mathrm{a}}$ & $806,48^{\mathrm{ab}}$ & $2859,89^{\mathrm{ab}}$ & $1550,66^{\mathrm{ab}}$ \\
\hline
\end{tabular}

Medias con una letra común no son significativamente diferentes $(\mathrm{p}>0,05)$ de acuerdo con la prueba LSD Fischer / Means with a common letter are not significantly different ( $\mathrm{p}>0.05)$ according to the LSD Fischer test.

ConH: con hojarasca; SinH: sin hojarasca; ConIP: con inóculo primario; SinIP: sin inóculo primario / ConH: with litterfall; SinH: without litterfall; ConIP: with primary inoculum; SinIP: without primary inoculum.

El análisis de varianza para cada factor no mostró diferencias significativas para ninguno de ellos en ninguna de las variables evaluadas en el año 2013, pero sí en el segundo año de evaluación (Cuadro 3). 
Cuadro 3. Valores de probabilidad de significación estadística para cada factor evaluado, hojarasca e inóculo primario, de acuerdo con el análisis de varianza del experimento para determinar el impacto de los factores sobre la epidemiología del ojo de gallo (Mycena citricolor) en café (Coffea arabica L. cultivar Catuaí). Tarrazú, Costa Rica. 2013-2014.

Table 3. Probability values of statistical significance for each evaluated factor, litterfall, and primary inoculum according to the variance analysis of the experiment to determine the impact of the factors on the epidemiology of the American leaf spot (Mycena citricolor) in coffee (Coffea arabica L. cultivar Catuaí). Tarrazú, Costa Rica. 2013-2014.

\begin{tabular}{|c|c|c|c|c|c|c|}
\hline \multirow[b]{3}{*}{ Factor } & \multicolumn{6}{|c|}{ Área bajo la curva de desarrollo de la enfermedad } \\
\hline & \multicolumn{3}{|c|}{2013} & \multicolumn{3}{|c|}{2014} \\
\hline & $\begin{array}{c}\text { Hojas } \\
\text { enfermas }\end{array}$ & Lesiones & $\begin{array}{c}\text { Geminíferos } \\
\text { activos }\end{array}$ & $\begin{array}{c}\text { Hojas } \\
\text { enfermas }\end{array}$ & Lesiones & $\begin{array}{l}\text { Geminíferos } \\
\text { activos }\end{array}$ \\
\hline Hojarasca & 0,4602 & 0,9317 & 0,1376 & 0,375 & 0,310 & 0,590 \\
\hline Inóculo Primario & 0,8413 & 0,9728 & 0,6002 & $0,013 *$ & $0,011^{*}$ & $0,006^{*}$ \\
\hline
\end{tabular}

* probabilidades estadísticamente significativas /* statistically significant probabilities.

En el primer año de estudio, los tratamientos sin remoción de inóculo inicial presentaron los mayores valores de crecimiento acumulado, mientras que todos los tratamientos se comportaron de forma muy similar en cuanto a la defoliación total del hospedero (Cuadro 4). No se encontraron diferencias estadísticamente significativas entre tratamientos para estas variables.

Cuadro 4. Área bajo la curva de desarrollo del crecimiento, la defoliación total y la defoliación de hojas enfermas de café (Coffea arabica L. cultivar Catuaí), causados por el ojo de gallo (Mycena citricolor). Tarrazú, Costa Rica. 2013-2014.

Table 4. Area under the growth development curve, total defoliation, and defoliation of diseased coffee leaves (Coffea arabica L. cultivar Catuaí) caused by American leaf spot (Mycena citricolor). Tarrazú, Costa Rica. 2013-2014.

\begin{tabular}{lcccccc}
\hline & \multicolumn{5}{c}{ Área bajo la curva de desarrollo } \\
\cline { 2 - 7 } Tratamiento & \multicolumn{5}{c}{$\mathbf{2 0 1 3}$} & \multicolumn{2}{c}{$\mathbf{2 0 1 4}$} \\
\cline { 2 - 6 } & Crecimiento & Defoliación & $\begin{array}{c}\text { Defoliación de } \\
\text { hojas enfermas }\end{array}$ & Crecimiento & Defoliación & $\begin{array}{c}\text { Defoliación de } \\
\text { hojas enfermas }\end{array}$ \\
\hline ConH/ConIP & $827,35^{\mathrm{a}}$ & $252,96^{\mathrm{a}}$ & $56,04^{\mathrm{a}}$ & $1188,64^{\mathrm{a}}$ & $342,27^{\mathrm{a}}$ & $44,36^{\mathrm{ab}}$ \\
ConH/SinIP & $814,80^{\mathrm{a}}$ & $220,84^{\mathrm{a}}$ & $41,30^{\mathrm{a}}$ & $1171,84^{\mathrm{a}}$ & $336,34^{\mathrm{a}}$ & $17,36^{\mathrm{a}}$ \\
SinH/ConIP & $823,66^{\mathrm{a}}$ & $229,38^{\mathrm{a}}$ & $46,87^{\mathrm{a}}$ & $1174,27^{\mathrm{a}}$ & $389,10^{\mathrm{a}}$ & $74,38^{\mathrm{b}}$ \\
SinH/SinIP & $795,64^{\mathrm{a}}$ & $227,50^{\mathrm{a}}$ & $51,84^{\mathrm{a}}$ & $1167,40^{\mathrm{a}}$ & $324,44^{\mathrm{a}}$ & $18,52^{\mathrm{a}}$ \\
\hline
\end{tabular}

Medias con una letra común no son significativamente diferentes $(\mathrm{p}>0,05)$ de acuerdo con la prueba LSD Fischer / Means with a common letter are not significantly different $(\mathrm{p}>0.05)$ according to the LSD Fischer test.

ConH: con hojarasca; SinH: sin hojarasca; ConIP: con inóculo primario; SinIP: sin inóculo primario / ConH: with litterfall; SinH: without litterfall; ConIP: with primary inoculum; SinIP: without primary inoculum.

De igual manera, para el segundo año de evaluación, todos los tratamientos se comportaron de forma muy similar en cuanto al crecimiento y la defoliación de las plantas; los valores de áreas bajo la curva de desarrollo de estas variables no fueron significativamente diferentes entre tratamientos (Cuadro 4). Sin embargo, se pudo notar que las plantas que presentaron mayor defoliación de hojas enfermas fueron las que estuvieron expuestas a los tratamientos con inóculo primario. 
El modelo de crecimiento logístico presentó los mayores coeficientes de determinación $\left(\mathrm{R}^{2}\right)$ y los menores cuadrados medios del error para ambos años de evaluación. No se encontró dispersión aleatoria al graficar los residuos estandarizados contra los valores de enfermedad predichos en ninguno de los análisis de regresión realizados para los modelos evaluados.

La tasa de infección $(r)$ de dicho modelo fue prácticamente la misma para todos los tratamientos en el primer año, 0,04 unidades por día; cambió levemente para la epidemia siguiente (Cuadro 5).

Cuadro 5. Resumen de los estadísticos del análisis de regresión lineal simple usados en la evaluación de la conveniencia de los modelos monomolecular, logístico y Gompertz, para describir el progreso del ojo de gallo (Mycena citricolor) en café (Coffea arabica L. cultivar Catuaí), en cada tratamiento de remoción de hojarasca e inóculo primario. Tarrazú, Costa Rica. 2013-2014.

Table 5. Summary of the simple linear regression statistics analysis used in the evaluation of the convenience of the monomolecular, logistic, and Gompertz models, to describe the progress of American leaf spot (Mycena citricolor) in coffee Coffea arabica L. cultivar Catuaí) in each treatment of litterfall removal and primary inoculum. Tarrazú, Costa Rica. 2013-2014.

\begin{tabular}{llcccccc}
\hline & & \multicolumn{3}{c}{$\mathbf{2 0 1 3}$} & & \multicolumn{2}{c}{$\mathbf{2 0 1 4}$} \\
\cline { 3 - 7 } Tratamiento & Modelo & $\mathbf{R}^{\mathbf{2}}$ & $\mathbf{r}$ & $\mathbf{Q}$ & $\mathbf{R}^{\mathbf{2}}$ & $\mathbf{r}$ & $\mathbf{0}$ \\
\hline ConH/ConIP & Monomolecular & 49,09 & 0,0291 & 0,0145 & 74,10 & 0,0319 & 0,0159 \\
& Logístico & 70,86 & 0,044 & 0,0073 & 82,15 & 0,0415 & 0,0069 \\
& Gompertz & 59,15 & 0,0351 & 0,0087 & 78,23 & 0,0362 & 0,0090 \\
ConH/SinIP & Monomolecular & 45,95 & 0,0276 & 0,0138 & 65,40 & 0,0255 & 0,0127 \\
& Logístico & 70,28 & 0,0437 & 0,0072 & 89,56 & 0,0553 & 0,0092 \\
& Gompertz & 57,08 & 0,0437 & 0,0109 & 78,77 & 0,0357 & 0,0089 \\
SinH/ConIP & Monomolecular & 51,31 & 0,0271 & 0,0135 & 73,29 & 0,0306 & 0,0153 \\
& Logístico & 75,79 & 0,0452 & 0,0075 & 79,71 & 0,0375 & 0,0062 \\
& Gompertz & 63,12 & 0,034 & 0,0085 & 76,59 & 0,0338 & 0,0084 \\
SinH/SinIP & Monomolecular & 36,58 & 0,0402 & 0,0201 & 74,69 & 0,0286 & 0,0143 \\
& Logístico & 70,26 & 0,0484 & 0,0080 & 91,82 & 0,0584 & 0,0097 \\
& Gompertz & 56,30 & 0,037 & 0,0092 & 84,49 & 0,0389 & 0,0097 \\
\hline
\end{tabular}

$\mathrm{R}^{2}$ : coeficiente de determinación (suma de cuadrados del error / cuadrado medio del error), r: tasa de infección aparente, @: parámetro Rho (tasa absoluta ponderada de incremento de la enfermedad) para comparación entre tasas de infección aparente de los diferentes modelos / $\mathrm{R}^{2}$ : coefficient of determination (sum of error squares / mean square error), r: apparent infection rate, @: Rho parameter (weighted absolute rate of disease increase) for comparison between apparent infection rates of the different models.

ConH: con hojarasca; SinH: sin hojarasca; ConIP: con inóculo primario; SinIP: sin inóculo primario / ConH: with litterfall; SinH: without litterfall; ConIP: with primary inoculum; SinIP: without primary inoculum.

Las correlaciones que contienen la variable cantidad total de geminíferos $(\mathrm{G})$ y promedio de geminíferos por lesión (GL), presentaron asociaciones significativas con la humedad relativa (HR) para ambos años de estudio epidemiológico (Cuadro 6).

Para el año 2014, las asociaciones de cantidad total de geminíferos (G) con las variables ambientales humedad relativa (HR), precipitación (PREC) y temperatura (TEMP) fueron significativas en todos los tratamientos. La cantidad de geminíferos activos $(\mathrm{G})$ correlacionó negativamente con la temperatura en todos los tratamientos (Cuadro 7).

La variable promedio de geminíferos por lesión (GL) correlacionó significativamente en tres de los cuatro tratamientos (ConH/ConIP, SinH/ConIP y SinH/SinIP) para su asociación con las variables humedad relativa (HR) y temperatura (TEMP) (Cuadro 6). 
Cuadro 6. Coeficientes de correlación de Spearman (rs), probabilidad (p) y significancia (Sig.) entre las condiciones ambientales y las variables evaluadas en el tratamiento con hojarasca y con inóculo primario (ConH/ConIP) (manejo tradicional) de remoción de hojarasca de café (Coffea arabica L. cultivar Catuaí) e inóculo primario de ojo de gallo (Mycena citricolor). Tarrazú, Costa Rica. 20132014.

Table 6. Spearman correlation coefficients (rs), probability (p), and significance (Sig.) between the environmental conditions and the variables in the treatment with litterfall and primary inoculum (ConH/ConIP) (traditional management) of coffee leaf (Coffea arabica L. cultivar Catuaí) litter removal and primary inoculum of American leaf spot (Mycena citricolor). Tarrazú, Costa Rica. $2013-2014$.

\begin{tabular}{|c|c|c|c|c|c|c|}
\hline \multirow[t]{2}{*}{ Asociaciones } & \multicolumn{3}{|c|}{2013} & \multicolumn{3}{|c|}{2014} \\
\hline & $r_{s}$ & $p$ & Sig. & $r_{s}$ & $p$ & Sig. \\
\hline HE*HR & 0,6571 & 0,1562 & ns & $-0,1261$ & 0,1726 & ns \\
\hline $\mathrm{HE}^{*} \mathrm{MF}$ & 0,8857 & 0,0188 & $* *$ & 0,5797 & 0,7876 & ns \\
\hline HE*PREC & 0,8857 & 0,0188 & $* *$ & $-0,2523$ & 0,5852 & ns \\
\hline HE*TEMP & $-0,8857$ & 0,0188 & $* *$ & $-0,0901$ & 0,8477 & ns \\
\hline $\mathrm{L} * \mathrm{HR}$ & 0,6571 & 0,1562 & ns & 0,2500 & 0,5887 & ns \\
\hline $\mathrm{L} * \mathrm{MF}$ & 0,8857 & 0,0188 & $* *$ & 0,7042 & 0,0774 & ns \\
\hline L*PREC & 0,8857 & 0,0188 & $* *$ & 0,000 & 1,000 & ns \\
\hline L*TEMP & $-0,8857$ & 0,0188 & $* *$ & $-0,4643$ & 0,2939 & ns \\
\hline $\mathrm{G}^{*} \mathrm{HR}$ & 0,9411 & 0,0051 & $* * *$ & 0,9286 & 0,0025 & $* * *$ \\
\hline $\mathrm{G}^{*} \mathrm{MF}$ & 0,8804 & 0,0206 & $* *$ & 0,4077 & 0,3639 & $\mathrm{~ns}$ \\
\hline G*PREC & 0,6983 & 0,1228 & $\mathrm{~ns}$ & 0,8571 & 0,0137 & $* *$ \\
\hline G*TEMP & $-0,6983$ & 0,1228 & ns & $-0,8929$ & 0,0068 & $* * *$ \\
\hline LH*HR & 0,6000 & 0,2080 & $\mathrm{~ns}$ & 0,2500 & 0,5887 & ns \\
\hline $\mathrm{LH}^{*} \mathrm{MF}$ & 0,7714 & 0,0724 & ns & 0,7042 & 0,0774 & ns \\
\hline LH*PREC & 0,9429 & 0,0048 & $* * *$ & 0,000 & 1,000 & ns \\
\hline LH*TEMP & $-0,9429$ & 0,0048 & $* * *$ & $-0,4643$ & 0,2939 & ns \\
\hline $\mathrm{GL} * \mathrm{HR}$ & 0,8452 & 0,0341 & $* *$ & 0,7857 & 0,0362 & $* *$ \\
\hline $\mathrm{GL} * \mathrm{MF}$ & 0,7775 & 0,0687 & $\mathrm{~ns}$ & 0,559 & 0,1950 & ns \\
\hline GL*PREC & 0,3719 & 0,4679 & $\mathrm{~ns}$ & 0,5357 & 0,2152 & ns \\
\hline GL*TEMP & $-0,3719$ & 0,4679 & $\mathrm{~ns}$ & $-0,8571$ & 0,0137 & $* *$ \\
\hline
\end{tabular}

ns: no estadísticamente significativo, $* *$ : estadísticamente significativo si $\mathrm{p}<0,05$, ***: altamente significativo si p<0,01, HE: cantidad total de hojas enfermas, L: cantidad total de lesiones, G: cantidad total de geminíferos activos, LH: promedio de lesiones por hoja, GL: promedio de geminíferos activos por lesión, HR: humedad relativa (\%), MF: mojadura foliar (horas), PREC: precipitación (mm), TEMP: temperatura (C) / ns: not statistically significant, **: statistically significant if $\mathrm{p}<0.05$, ***: highly significant if $\mathrm{p}<0.01$, HE: total number of diseased leaves, L: total number of lesions, G: total number of active geminifers, LH: average of lesions per leaf, GL: average of active geminifers per lesion, HR: relative humidity (\%), MF: foliar wetting (hours), PREC: precipitation (mm), TEMP: temperature $(\mathrm{C})$.

\section{Discusión}

El ojo de gallo en café no superó el $25 \%$ de incidencia en ninguno de los años de estudio, debido, probablemente, a que las condiciones ambientales fueron atípicas para la zona y poco conducentes para la enfermedad. Durante el período evaluado la precipitación anual acumulada no superó el mínimo para una zona lluviosa, sobre todo en el año 2013 que contabilizó $500 \mathrm{~mm}$ de lluvia menos que el mínimo para el sitio. Además, las temperaturas mínimas registradas estuvieron hasta $6{ }^{\circ} \mathrm{C}$ por encima del mínimo normal para esta región, condiciones que desfavorecieron el desarrollo del ojo de gallo, pero que fueron suficientes para el progreso de la roya, enfermedad que disminuye su período de incubación conforme aumenta la temperatura (Ribeiro et al., 1978). 
Cuadro 7. Coeficientes de correlación de Spearman para las asociaciones de condiciones ambientales con total de geminíferos y geminíferos por lesión por tratamiento del experimento de remoción de hojarasca de café (Coffea arabica L. cultivar Catuaí) e inóculo primario de ojo de gallo (Mycena citricolor). Tarrazú, Costa Rica. 2014.

Table 7. Spearman correlation coefficients for the associations of environmental conditions with total geminifers and geminifers due to injury due to treatment of the coffee (Coffea arabica L. cultivar Catuaí) litterfall removal and primary inoculum experiment of the American leaf spot (Mycena citricolor) experiment. Tarrazú, Costa Rica. 2014.

\begin{tabular}{|c|c|c|c|c|c|c|c|c|}
\hline \multirow{3}{*}{$\begin{array}{l}\text { Asociacio- } \\
\text { nes }\end{array}$} & \multicolumn{8}{|c|}{ Tratamiento } \\
\hline & \multicolumn{2}{|c|}{ ConH/ConIP } & \multicolumn{2}{|c|}{ ConH/SinIP } & \multicolumn{2}{|c|}{ SinH/ConIP } & \multicolumn{2}{|c|}{ SinH/SinIP } \\
\hline & $r_{s}$ & $p$ & $r_{s}$ & $p$ & $r_{s}$ & $p$ & $r_{s}$ & $p$ \\
\hline G*HR & 0,9286 & $0,0025 * * *$ & 0,9286 & $0,0025 * * *$ & 0,9643 & $0,0005 * * *$ & 0,9643 & $0,0005 * * *$ \\
\hline $\mathbf{G}^{*} \mathbf{M F}$ & 0,4077 & $0,3639^{\text {ns }}$ & 0,4077 & $0,3639^{\text {ns }}$ & 0,2965 & $0,5185^{\mathrm{ns}}$ & 0,2965 & $0,5185^{\mathrm{ns}}$ \\
\hline G*PREC & 0,8571 & $0,0137 * *$ & 0,8571 & $0,0137 * *$ & 0,8929 & $0,0068 * * *$ & 0,8929 & 0,0068 *** \\
\hline G*TEMP & $-0,8929$ & $0,0068 * * *$ & $-0,8929$ & $0,0068 * * *$ & $-0,9286$ & $0,0025 * * *$ & $-0,9286$ & $0,0025 * * *$ \\
\hline GL*HR & 0,7857 & $0,0362 * *$ & 0,6071 & $0,1482^{\mathrm{ns}}$ & 0,8571 & $0,0137 * *$ & 0,8571 & $0,0137 * *$ \\
\hline GL*TEMP & $-0,8571$ & $0,0137 * *$ & $-0,7143$ & $0,0713^{\text {ns }}$ & $-0,9286$ & $0,0025 * *$ & $-0,9286$ & 0,0025 *** \\
\hline
\end{tabular}

ns: no estadísticamente significativo, $* *$ : estadísticamente significativo si $\mathrm{p}<0,05, * * *$ : altamente significativo si $\mathrm{p}<0,01, \mathrm{G}$ : cantidad total de geminíferos activos, GL: promedio de geminíferos activos por lesión, HR: humedad relativa (\%), MF: mojadura foliar (horas), PREC: precipitación (mm), TEMP: temperatura (C) / ns: not statistically significant, $* *$ : statistically significant if $\mathrm{p}<0.05$, $* * *$ : highly significant if $\mathrm{p}<0.01, \mathrm{G}$ : total number of active geminifers, GL: average of active geminifers per lesion, HR: relative humidity (\%), MF: foliar wetting (hours), PREC: precipitation (mm), TEMP: temperature (C).

ConH: con hojarasca; SinH: sin hojarasca; ConIP: con inóculo primario; SinIP: sin inóculo primario / ConH: with litterfall; SinH: without litterfall; ConIP: with primary inoculum; SinIP: without primary inoculum.

Desde el año 2012 todos los países centroamericanos, el Caribe y México, sufrieron fuerte afectación por una epidemia de roya anaranjada (Hemileia vastatrix Berk. \& Broome) (Avelino y Rivas, 2013).

Debido a que las condiciones atípicas de temperatura y precipitación afectaron el desarrollo de las epidemias, en el 2013 (año con mayor déficit de lluvia) no fue posible encontrar efecto de $y_{0}$; mientras que sí fue posible observar diferencias bajo las condiciones del 2014, año en el que se registraron $500 \mathrm{~mm}$ de lluvia por encima del anterior, lo que permitió un mejor desarrollo del ojo de gallo en la zona.

Al revisar las asociaciones entre los descriptores de la enfermedad y las condiciones ambientales, se notó que la cantidad total de geminíferos (G) y el promedio de geminíferos por lesión (GL) correlacionaron positiva y significativamente con la humedad relativa, $\mathrm{R}^{2}=0,94$ y $\mathrm{R}^{2}=0,84$, respectivamente; así como con la cantidad de horas de mojadura foliar para la primera variable $\left(\mathrm{R}^{2}=0,88\right)$. Además, las variables $\mathrm{G}$ y PREC (precipitación) presentaron un $\mathrm{R}^{2}=0,69$. De acuerdo con esto, muy probablemente las variables ambientales mencionadas determinaron la aparición de geminíferos en las lesiones. Esto se confirma con los resultados obtenidos por Vargas (2004), quien desarrolló una ecuación de predicción con cinco días de anticipación para la variable de lesiones con cuerpos geminíferos a partir de los valores de humedad relativa y mojadura foliar, de esta forma con un $\mathrm{R}^{2}=0,67$ estableció la ecuación $y=0,334-0,224 * M F+0,002\left(H R^{*} M F\right)$ que explicó la variación de lesiones con estructuras de ojo de gallo. Este comportamiento se ajusta a lo mencionado por Staver et al. (2001), quienes indicaron que enfermedades producidas por M. citricolor, Phoma costarricensis Echandi y Corticium koleroga (Cooke) Höhn. (como Pellicularia koleroga Cooke) se correlacionan positiva y uniformemente con la humedad.

El crecimiento del hospedero fue similar en todos los tratamientos, lo que presume que las condiciones propias de la planta no tuvieron ningún efecto sobre el desarrollo de la enfermedad, y los efectos se debieron a los tratamientos aplicados. 
Con respecto a la defoliación total, sumatoria de la caída de hojas enfermas y sanas, o al menos sin enfermedad aparente, se mantuvo muy similar para todos los tratamientos. La defoliación de hojas con ojo de gallo se comportó de manera muy similar a la incidencia, lo que supone que las plantas que mantuvieron su inóculo inicial estuvieron más propensas a eliminar las hojas enfermas durante el ciclo de la enfermedad.

Los resultados obtenidos concuerdan con lo dicho por varios investigadores, los cuales indicaron que el desarrollo de la epidemia depende de la fluctuación estacional de la lluvia y la humedad relativa (Wang y Avelino, 1999) y que la humedad se mantenga superior al 80 \% (Barquero, 2007). En Costa Rica, la máxima infección se presenta entre setiembre y octubre, que son los meses de mayor precipitación, y empieza a reducir en diciembre (Wang y Avelino, 1999). De acuerdo con Barquero (2012), durante estos meses se deben acumular $400 \mathrm{~mm}$ de lluvia en tres semanas consecutivas para que se presente la enfermedad.

La tasa de infección aparente hallada en esta investigación fue mayor a las reportadas por Wang y Arauz (1999) de 0,015 unidades día ${ }^{-1}$ y por Vargas (2004) de 0,004 unidades día ${ }^{-1}$, aunque sigue siendo baja para una enfermedad de tipo policíclica.

Si se cotejan los valores de $r$ típicos para enfermedades policíclicas, por ejemplo 0,12 unidades día ${ }^{-1}$ para roya anaranjada del cafeto (Kushalappa y Ludwing, 1982), con los valores de $r$ para ojo de gallo reportados previamente y en esta investigación, el ojo de gallo se asemeja a una enfermedad de tipo monocíclica.

La influencia del inóculo primario puede variar en las enfermedades de ciclo múltiple en relación con el valor de $r$; de esta forma el nivel de inóculo es importante si la $r$ es baja (Nutter, 2007; Arauz, 2011), como en el caso del ojo de gallo. Cuando $r$ es baja el desarrollo de la epidemia disminuye, significativamente, al reducir la cantidad de inóculo inicial; así, para una enfermedad con $r$ de 0,02 se puede retrasar la epidemia once días si se logra disminuir el inóculo inicial en $20 \%$, con $50 \%$ de reducción de inóculo inicial el retraso puede llegar a 35 días y con el 80 $\%$ se puede llegar hasta 80 días de retraso (Nutter, 2007). Sin embargo, con los datos hallados en las epidemias del 2013 no se logró el efecto de disminución de enfermedad al eliminar el $100 \%$ del inóculo primario. Es difícil determinar las causas de este comportamiento, casi todos los factores bióticos y abióticos pueden influenciar la tasa de infección aparente (Nutter, 2007). Es posible que la mayor fuente de inóculo primario para el ojo de gallo no esté constituida por las lesiones presentes en la planta al inicio de la estación lluviosa, sino por el inóculo que se encuentra en otras fuentes, hospedantes alternos, tales como malezas y árboles de sombra.

La forma de las curvas de desarrollo de la enfermedad para el año 2014 se asemejan en gran medida a la curva obtenida por Vargas et al. (1990), con nivel de enfermedad inicial $\left(y_{0}\right)$ bajo. Estos investigadores encontraron disminución en el desarrollo de la enfermedad en una epidemia de ojo de gallo evaluada en 1985 en condiciones de $y_{0}$ menor del $10 \%$, en comparación con $y_{0}$ superior al $70 \%$. Para ellos, la curva de la enfermedad con mayor nivel inicial se mantuvo por encima del $80 \%$ durante todo el período lluvioso, mientras que el desarrollo de la enfermedad con menor $y_{0}$ fue menor durante todo el período, para los meses de junio, julio y agosto se notó una diferencia del $60 \%$ entre una curva y otra; a partir de setiembre la diferencia entre ambas enfermedades bajó al $30 \%$, manteniéndose siempre más enfermas las plantas que iniciaron con mayor enfermedad; al final de la época lluviosa la diferencia se redujo a un valor aproximado del $5 \%$. Esos resultados confirman la importancia de $y_{0}$ en enfermedades policíclicas con tasa de infección baja.

En esta investigación la diferencia entre enfermedad inicial, en tratamientos con y sin eliminación de inóculo primario, fue solamente del $7 \%$ como máximo, esto no permitió cuantificar diferencias en el desarrollo de la enfermedad con la reducción de $y_{0}$ como las encontradas por Vargas et al. (1990).

Parece que el efecto de la remoción de la hojarasca no estuvo relacionado con la cantidad de inóculo que pueda existir en ella; ya que, bajo el supuesto de que $M$. citricolor tiene un hábito de vida saprófito y descomponedor de hojarasca, se debió encontrar diferencias en los niveles de enfermedad con los tratamientos de remoción de hojarasca. Sin embargo, en los dos años de estudio, fueron los tratamientos sin hojarasca los que desarrollaron mayor enfermedad en términos absolutos; esto sugiere que la hojarasca no tuvo un rol importante como fuente de inóculo del ojo de gallo, lo cual refuerza las observaciones realizadas por Wellman (1951), quien mencionó que los 
propágulos que caen sobre el suelo o materia en descomposición no sobreviven en el campo, ya que requieren de una reserva continua de hojas vivas.

\section{Conclusiones}

La remoción de la hojarasca presente en el suelo del cafetal no impactó significativamente la epidemia de ojo de gallo bajo las condiciones de este estudio; contrario al inóculo presente en las hojas adheridas a las plantas, que sí influyó en el desarrollo de la enfermedad. Este trabajo establece la primera investigación que trata de determinar el impacto de posibles fuentes de inóculo distintas a las lesiones presentes en hojas de café.

Se sugiere investigar acerca del impacto de hospedantes alternos en la epidemiología del ojo de gallo.

\section{Agradecimientos}

Los autores expresan su agradecimiento al Instituto Earthwatch por el financiamiento de la investigación mediante el proyecto "Costa Rican coffee from community to cup", gratitud especial a la cooperativa de caficultores de Santa María de Dota (Coopedota R.L) y al Centro Agrícola Cantonal de Tarrazú, por permitir usar sus fincas para el establecimiento de los ensayos, particularmente a los ingenieros Daniel Ureña y Rodrigo Jiménez.

\section{Literatura citada}

Achicanoy, H. 2000. Descripción cuantitativa de las epidemias de las plantas. Rev. Fac. Nac. Agron. Medellín 53:941-968.

Agrios. G. 2005. Plant pathology. $5^{\text {th }}$ ed. Academic Press, Cambridge, MA, USA.

Arauz, L.F. 2011. Fitopatología: un enfoque agroecológico. 2da ed. Editorial de la Universidad de Costa Rica, San José, CRI.

Avelino, J., y G. Rivas. 2013. La roya anaranjada del cafeto. HALL archive-ouvertes, FRA. https://hal.archives-ouvertes.fr/hal01071036/file/LA_ROYA_ANARANJADA_DEL_CAFETO_V1.pdf (consultado 19 mar. 2019).

Avelino, J., J.C. Toledo, y B. Medina. 1995. Desarrollo del ojo de gallo (Mycena citricolor) en una finca del norte de Guatemala y evaluación de los daños provocados por esta enfermedad. En: IICA-PROMECAFE, editores, XVI Simposio de Caficultura Latinoamericana. IICA-PROMECAFE, Managua, NIC. p. 1-8.

Barquero, M. 2007. Algunas consideraciones sobre el ojo de gallo. Rev. Infor. Icafe 1:11-15.

Barquero, M. 2010a. Más de 75.000 fanegas de café se perdieron por 'ojo de gallo'. La Nación, CRI. https://www.nacion.com/ economia/mas-de-75-000-fanegas-de-cafe-se-perdieron-por-ojo-de-gallo/N6HUXV5ALZBBTPJQLCBEIFZ3V4/story/ (consultado 13 may. 2011).

Barquero, M. 2010b. Cafetaleros perdieron $12 \%$ de cosecha por lluvia y plagas. La Nación, CRI. https://www.nacion. com/economia/cafetaleros-perdieron-12-de-cosecha-por-lluvia-y-plagas/6NKK6NCILRDDXESMW5PN7PURJY/story/ (consultado 13 may. 2011).

Barquero, M. 2012. Sistema de alerta temprana para el ojo de gallo. Rev. infor. Icafe 2012(2):2-4.

Borbón, O. 1999. Consideraciones sobre la problemática del ojo de gallo (Mycena citricolor) en Costa Rica. 1999. En: Colegio de Ingenieros Agrónomos, editores, "Memoria” XI Congreso Agronómico /IV Congreso Nacional de Fitopatología. Colegio de Ingenieros Agrónomos, CRI. p. 21-25.

Buller, A.H. 1958. Omphalia flavida, a gemmiferous and luminous leaf-spot fungus. In: A.H. Buller, editor, Researches on fungi. Vol. 6. Hafner Publishing Co., NY, USA. p. 397-454. 
Cannon, P.F., and P.M. Kirk. 2007. Fungal families of the world. CABI Publishing, Wallingford, GBR. doi: $10.1079 / 9780851998275.0000$

Carvajal, F. 1939a. Ojo de gallo (Omphalia flavida). Rev. Inst. Defensa Café 52:535-549.

Carvajal, F. 1939b. Ojo de gallo (Omphalia flavida): Segunda parte, la forma perfecta del hongo "Omphalia flavida" creciendo libremente en la naturaleza como parásito sobre plantas vivas, en el distrito cafetalero del Zapote (Prov. de San José, CR). Rev. Inst. Defensa Café 52:550-565.

COENOS (Comisión Técnica Consultiva Nacional del Fenómeno ENOS). 2010. Posible fenómeno de La Niña. Boletín del ENOS 33:1-6.

Dennis, R.W.G. 1961. Fungi venezuelani: IV. Agaricales. Kew Bull. 15:67-156. doi:10.2307/4115784

Di-Rienzo, J.A., F. Casanoves, M.G. Balzarini, L. González, M. Cuadroda, y C.W. Robledo. 2015. InfoStat Software estadístico. Grupo InfoStat, Universidad Nacional de Córdoba, ARG. http://www.infostat.com.ar (consultado 5 nov. 2015).

Guerra, B. 2004. Experiencias del manejo de las enfermedades fungosas presentes en el cultivo de café (Coffea arabica) en finca de pequeños caficultores en la aldea La Montaña, Moyuta, Jutiapa. Tesis Lic., Universidad de San Carlos, San Carlos, GTM.

Hernández, I. 2009. Diversidad de macrohongos de hojarasca en el bosque estatal de Guajataca. Tesis M.Sc., Universidad del Turabo, PRI.

Hibbett, D., and R. Thorn. 2001. Basidiomicotina: holobasidiomicetes. In: D. McLaughlin et al., editors, The mycota. VII Part A. Systematics and evolution. Springer-Verlag Berlin Heidelberg, NY, USA. p. 1-366.doi:10.1007/978-3-662-10189-6_5

Holb, I.J. 2006. Effect of six sanitation treatments on leaf litter density, ascospore production of Venturia inaequalis and scab incidence in integrated and organic apple orchards. Eur. J. Plant Pathol. 115:293-307. doi:10.1007/s 10658-006-9013-8

ICAFE (Instituto del Café de Costa Rica). 2019. Tarrazú. ICAFE, CRI. http://www.icafe.cr/nuestro-cafe/regiones-cafetaleras/ tarrazu/ (consultado 12 set. 2019).

Konan, J.K., and D.I. Guest. 2002. Leaf litter mulch reduces the survival of Phytophthora palmivora under cocoa trees in Papua New Guinea. Australas. Plant Pathol. 31:381-383. doi:10.1071/AP02043

Kushalappa, A., and A. Ludwig. 1982. Calculation of apparent rate in plant disease: development of method to correct for host growth. Phytopathology 72:1373-1377.

Moncalvo, J.M., R. Vilgalys, S. Redhead, J.E. Johnson, T. James, M.C. Aime, V. Hofstetter, S. Verduin, E. Larsson, T.J. Baroni, G. Thorn, S. Jacobsson, H. Clemencon, and O.K. Miller. 2002. One hundred and seventeen clades of euagarics. Mol. Phylogen. Evol. 23:357-400. doi:10.1016/s1055-7903(02)00027-1

Nutter, F.W. 2007. The role of plant disease epidemiology in developing successful integrated disease management programs. In: A. Ciancio, and K.G. Mukerji, editors, General concepts in integrated pest and disease management. Springer, Dordrecht, NED. p. 45-79. doi:10.1007/978-1-4020-6061-8_3

Plá, J.L. 1952. Informe sobre deshoja parcial del café. Suelo Tico 28:209-213.

Riberio, I.J., L.C. Monaco, O. Tisseli-Filho, e M.H. Sugimori. 1978. Efeito de alta temperatura no desenvolvimento de Hemileia vastatrix em caffeiro susceptivel. Bragantia 37(2):11-16. doi:10.1590/S0006-87051978000100002

Solano, J., y R. Villalobos. sf. Regiones y subregiones climáticas de Costa Rica. IMN, CRC. https://www.imn.ac.cr/ documents/10179/20909/Regionalización+climática+de+Costa+Rica (consultado 2 may. 2015).

Staver, C., F. Guharay, D. Monterroso, and R.G. Muschler. 2001. Designing pest-suppressive multistrata perennial crop systems: shade-grown coffee in Central America. Agrofor. Syst. 53:151-170. doi:10.1023/a:1013372403359 
Vargas, L.G. 2004. Bases epidemiológicas para el desarrollo de un sistema de pronóstico en ojo de gallo (Mycena citricolor Berk. y Curt) Sacc. en cafeto (Coffea arabica). Tesis M.Sc., Universidad de Costa Rica, San José, CRI.

Vargas, E., M. González, G. Umaña, y L. Vargas. 1990. Nuevas alternativas de combate químico del ojo de gallo (Mycena citricolor). En: IICA/PROMECAFE, editores, XII Simposio sobre Caficultura Latinoamericana. IICA, CRI. p. 425.

Wang, A., y L.F. Arauz. 1999. Aplicación de principios epidemiológicos para el combate de ojo de gallo en cafeto. En: Colegio de Ingenieros Agrónomos, editores, "Memoria" XI Congreso Agronómico /IV Congreso Nacional de Fitopatología. Colegio de Ingenieros Agrónomos, CRI. p.9-12.

Wang, A., y J. Avelino. 1999. El ojo de gallo (Mycena citricolor). En: B. Bertrand, y B. Rapidel, editores, Desafíos de la caficultura en Centroamérica. IICA, San José, CRI. p. 243-260.

Webster, J., and R. Weber. 2007. Introduction to Fungi. 3rd ed. Cambridge University Press, Cambridge, GBR. doi:10.1017/ cbo9780511809026

Wellman, F.L. 1951. Control del ojo de gallo, Omphalia flavida, por medio de la deshoja de cafetos enfermos (1). Trad. M. Gutiérrez, y J.M. Montero. Suelo Tico 24:42-51. 